\title{
Suppression of tumor invasion and migration in breast cancer cells following delivery of siRNA against Stat3 with the antimicrobial peptide PR39
}

\author{
WENXIA TIAN $^{1 *}$ BING LI $^{2 *}$, XIWEN ZHANG ${ }^{1}$, WEIQI DANG ${ }^{1}$, XIAOFEI WANG ${ }^{1}$, \\ HAO TANG $^{1}$, LIN WANG $^{1}$, HONG CAO $^{1}$ and TINGMEI CHEN ${ }^{1 *}$ \\ ${ }^{1}$ Department of Laboratory Medicine, Key Laboratory of Diagnostic Medicine, Ministry of Education, Chongqing \\ Medical University; ${ }^{2}$ Dean's Office of Chongqing Medical University, Chongqing 400016, P.R. China
}

Received April 11, 2012; Accepted May 25, 2012

DOI: $10.3892 /$ or.2012.1911

\begin{abstract}
PR39, a porcine cathelicidin rich in the amino acids proline and arginine can interact with the negatively charged component of the cell surface, and rapidly penetrate cell membranes. Therefore, we hypothesized that PR39, as a membrane penetrating peptide (MPP), could be exploited as a novel carrier to deliver siRNA into the cell cytoplasm in order to knockdown target gene expression. Firstly, a complex formation of PR39 with siRNA and its cellular colocalization were investigated in our studies. Further, we optimized the ratio of the PR39/siRNA complex, cell/complex incubation period and the concentration of siRNA. The results suggest that PR39 could form a complex with siRNA, and mediate translocation of the siRNA into 4T1 cells. The optimal ratio of siRNA with PR39 was 1:90 which was found to have a maximum Stat 3 gene silencing effect after $48 \mathrm{~h}$ treatment. Moreover, 4T1 cell proliferation, cell cycle, invasion and migration were investigated. The results suggested that Stat 3 knockdown could not result in 4T1 cell proliferation inhibition and cell cycle arrest, while invasion and migration of 4T1 cells were strongly inhibited. Notably, the data also showed that in addition to inhibition of carcinogenesis, single PR39 may play a role in cell invasion and migration. PR39 and Stat3 siRNA displayed synergistic biological effects in inhibiting cell invasion and migration of 4T1 cells, which was more prominent compared with the popular Lipofectamine delivery system.
\end{abstract}

Correspondence to: Professor Tingmei Chen, Department of Laboratory Medicine, Key Laboratory of Diagnostic Medicine Ministry of Education, Chongqing Medical University, 1 Yixueyuan Road, Yu Zhong District, Chongqing 400016, P.R. China

E-mail: chentingmei@sohu.com

${ }^{*}$ Contributed equally

Key words: porcine cathelicidin, signal transducers and activators of transcription-3, siRNA, antimicrobial peptide, matrix matalloproteinase-9

\section{Introduction}

The incidence and mortality of breast cancer has been rising in women over the past 15 years, and breast cancer has ranked first in women in China (1). Most of the treatment failures and deaths of breast cancer, particularly the more invasive hormone-dependent cases, were caused by tumor metastasis (2). Signal transducer and activator of transcription-3 (Stat3) is known to have frequently been aberrantly activated in a large percentage of breast cancers, and has been involved in numerous tumor biological processes, including cell proliferation, survival, tumor angiogenesis, invasion and migration $(3,4)$. Evidences supports that selectively suppressing constitutive Stat 3 signaling is an effective approach in inhibiting cancer-associated processes and cancer progression $(5,6)$. It has been reported that primary breast tumor growth and brain metastasis could be suppressed by Stat 3 inhibition (7). The established strategies targeting Stat 3 include tyrosine kinase inhibitors (e.g. tyrphostin AG490), antisense oligonucleotides, decoy oligonucleotides and dominant negative Stat 3 protein (8-13). In recent years, gene silencing by small interference RNA (siRNA) is a well-known and promising approach used to repress target gene expression $(14,15)$. However, as it is prone to degradation and has poor cellular permeability, naked siRNA cannot enter the intracellular environment where biological function of siRNA molecules occurs. Recently, various siRNA-delivery systems, liposomes, polymers, peptides (16) and membrane penetrating peptides (MPPs) have been highlighted. MPPs rich in basic amino acids, such as arginine and lysine, key motifs for the efficient delivery of extracellular molecules into cells are attractive peptide-mediated delivery systems (17).

PR39, a porcine cathelicidin is rich in proline $(49 \%)$ and arginine (26\%) and has been shown to be involved in antimicrobial activities (18), suppressing DNA and protein synthesis $(19,20)$. In addition to versatile biological functions, there is evidence that PR39 could penetrate cell membranes rapidly and interact with the SH3 domains of p47 phox $(21)$, p130 Cas (22), and the $\alpha 7$ subunit of the $20 \mathrm{~S}$ proteasome (23) because of its proline and arginine rich composition. Therefore, we hypothesized that PR39 could be exploited as a novel MMP, 
a carrier which would deliver siRNA into cells cytoplasm to knock down target gene expression.

In this study, we utilized PR39 to deliver siRNA selectively silencing Stat 3 in a mouse breast cancer cell line, 4T1, to repress Stat 3 expression as well as downstream components of the Stat 3 pathway. To our knowledge, this study provides the first evidence that PR39, as a vector, plays an important role in delivery of siRNA into 4T1 cells.

\section{Materials and methods}

Synthesis of PR39 and siRNA. Full length PR39 (RRRPR PPYLPRPRPPPFFPPRLPPRIPPGFPPRFPPRFP-NH $\mathrm{N}_{2}$ and FITC-PR39 (FITC-RRRPRPPYLPRPRPPPFFPPRLPPR IPPGFPPRFPPRFP-NH $\mathrm{N}_{2}$ ) were prepared by Shanghai Sangon Biological Engineering Technology and Services Corporation (Shanghai, China). The synthetic peptide PR39 was at least 95\% pure by high performance liquid chromatography (HPLC). PR39 was dissolved in phosphate-buffered saline (PBS, pH 7.4) to appropriate concentrations (4 mM). The small interference RNA oligo used for Stat 3 gene silencing (sense: 5'-GGACGACUUUGAUUUCAACtt-3', antisense: 5'-GUU GAAAUCAAAGUCGUCCtg-3') (24) and negative control (sense: 5'-UUCUCCGAACGUGUCACGUTT-3', antisense: 5'-ACGUGACACGUUCGGAGAATT-3') were purchased from Shanghai GenePharma Co., Ltd. (Shanghai, China). 5'Cy3-labeled siRNA was ordered from Guangzhou Ribobio Co., Ltd. (Guangzhou, China). The siRNA was dissolved in sterilized distilled $\mathrm{H}_{2} \mathrm{O}$ to the concentration of $20 \mu \mathrm{M}$.

Cell lines and antibodies. The 4T1 cells supplied by our laboratory were cultured in Dulbecco's modified Eagle's medium (DMEM, high glucose, Gibco, USA) with $10 \%$ fetal bovine serum (HyClone, USA) at $37^{\circ} \mathrm{C}$ in a $5 \% \mathrm{CO}_{2}$ atmosphere (Heraeus, Germany). Anti-Stat 3 was purchased from BectonDickinson, USA; anti-MMP-9 was obtained from Bioworld (USA); anti- $\beta$-actin was from Santa Cruz Biotechnology, Inc. (USA); HRP-conjugated secondary antibody was purchased from Zhongshan Golden Bridge Biotechnology Co., Ltd. (China).

Preparation of PR39/siRNA complex and gel shift assay. The Stat3 scrambled siRNA was diluted with appropriate PBS to a concentration of $100 \mathrm{nM}$, and PR39 was added into PBS. To determine the optimal relative concentration at which the PR39/siRNA complex formation would be advanced, $100 \mathrm{nM}$ of siRNA was incubated with different amounts of PR39 at $25^{\circ} \mathrm{C}$ for $15 \mathrm{~min}$ in PBS, with the siRNA/PR39 concentration ratio ranging from $0: 1$ to $1: 90$. The $\mathrm{Scr}$ siRNA/PR39 complexes were detected by electrophoresis on a $2 \%$ agarose gel (Invitrogen Life Technologies, USA) with GoldenView in TBE buffer at $180 \mathrm{~V}$ for $15 \mathrm{~min}$. Then, stained bands were visualized under UV light and photographed using the Bio-Rad Chemi Doc XRS imaging system (Bio-Rad, USA).

Observation of labeled PR39/siRNA complexes by fluorescence microscope. 4T1 cells were seeded on 10-mm cover slips with $5 \times 10^{4}$ cells/cover slip $18 \mathrm{~h}$ before transfection with the 5'Cy3-siRNA. The 5'Cy3-siRNA $(2.5 \mu \mathrm{l})$ was diluted into $50 \mu \mathrm{l}$ serum-free DMEM. PR39 (1.125 $\mu \mathrm{l})$ was diluted in $50 \mu \mathrm{l}$ serum-free DMEM, mixed gently and incubated for $5 \mathrm{~min}$ at room temperature. 5'Cy3-siRNA was combined with the diluted PR39. They were mixed gently and incubated for $30 \mathrm{~min}$ at room temperature to form a complex. This complex was added to the cells with $400 \mu \mathrm{l}$ serum-free DMEM. Then 4T1 cells were rinsed thrice with PBS after $6 \mathrm{~h}$ of transfection and fixed in methanol for $20 \mathrm{~min}$ at $-20^{\circ} \mathrm{C}$. The cells were washed twice with PBS and DAPI fluorescent stain (Beyotime, China) which was applied to the cells for $5 \mathrm{~min}$ at room temperature. The cells were rinsed three times with PBS and observed under a fluorescence microscope (Nikon, Japan).

Transient transfection of $4 T 1$ cells with Stat3 siRNA. Stat3 siRNA and scrambled control were transfected with PR39 (concentration ratio 1:90) or Lipofectamine 2000 reagent (Lipo 2000; Invitrogen, USA). All steps were performed following the manufacturer's instructions. The 4T1 cells suspended in complete DMEM medium were seeded at $6 \times 10^{5}$ cells/well in 6-well plates (Costar, USA) and allowed to attach overnight before transfection. The siRNA $(10 \mu \mathrm{l})$ was diluted into $250 \mu \mathrm{l}$ serum-free DMEM. PR39 $(4.5 \mu \mathrm{l})$ or Lipo $2000(5 \mu \mathrm{l})$ was diluted in $250 \mu \mathrm{l}$ serum-free DMEM, mixed gently and incubated for $5 \mathrm{~min}$ at room temperature. Then, Stat3 siRNA was combined with the diluted PR39 or Lipo 2000. They were mixed gently and incubated to form a complex for $30 \mathrm{~min}$ at room temperature. Then the complex was added to the cells with $1500 \mu \mathrm{l}$ serum-free DMEM. After $6 \mathrm{~h}$ transfections, cells were rinsed with PBS and the medium was replaced with fresh complete growth medium. The cells were incubated at $37^{\circ} \mathrm{C}$ in a $5 \% \mathrm{CO}_{2}$ atmosphere, and further analysis was performed 24,48 or $72 \mathrm{~h}$ post-transfection.

Cell viability assay. 4T1 cells were seeded at a density of $6 \times 10^{3} /$ well into 96-well plates (Costar, USA) and treated with various concentrations of PR39 or various treatment combinations for $24 \mathrm{~h}$. The next day, cells were incubated in methylthiazole tetrazolium (MTT, Sigma, USA) solution for $4 \mathrm{~h}$. The spectrophotometric absorbance was measured in a microplate reader (Bio-Rad) at $490 \mathrm{~nm}$. Absorbance rates obtained by untreated cells were considered as $100 \%$ cell survival. The relative cell viability was calculated according to the equation $\left(\mathrm{Abs}_{\text {sample }}{ }^{-}\right.$ $\left.\mathrm{Abs}_{\text {blank }}\right) /\left(\mathrm{Abs}_{\text {control }}-\mathrm{Abs}_{\text {blank }}\right) \times 100$. Each assay was repeated at least three times, using three wells per drug concentration in each experimental condition.

Flow cytometry. About $5 \times 10^{5} 4 \mathrm{~T} 1$ cells/well were seeded in 6 -well plate before various treatments. The Stat3-specific siRNA complex was added to the $4 \mathrm{~T} 1$ cells as above. The 4T1 cells from the control or treated group were trypsinized and suspended in $70 \%$ ethanol at $4^{\circ} \mathrm{C}$ at least overnight. Then samples were kept on ice and analyzed on a FACSCalibur flow cytometer (Becton-Dickinson).

RNA extraction and reverse transcription-polymerase chain reaction $(R T-P C R)$. Total cellular RNA was extracted by the Trizol Reagent (Takara, Japan) according to the manufacturer's instructions. Then, cDNA was obtained from the cellular RNA. For the reverse transcriptase reaction, $1 \mu \mathrm{g}$ of total RNA was mixed with oligo(dt) 15 primer and Maloney murine leukemia virus (M-MLV) reverse transcriptase (Takara). Then 
the mixture was incubated at $42^{\circ} \mathrm{C}$ for $60 \mathrm{~min}$ and $70^{\circ} \mathrm{C}$ for 15 min. The sequences of PCR primers (Invitrogen, China) were: Stat 3 forward, 5'-AGAGAAGCAGCAGATGTTGG AGCA-3', and reverse, 5'-ATCCTGCATGTCTCCTTGGCT CTT-3' (PCR product, 148 bp) (25); MMP-9 forward, 5'-ACAG CCAACTATGACCAG-3' and reverse; 5'-TGCCACCAGGA ACAGG-3' (PCR product, $249 \mathrm{bp}$ ). $\beta$-actin forward, 5 '-CCA CTGCCGCATCCTCTTCCTC-3', and reverse, 5'-TCCTGCTT GCTGATCCACATCT-3' (PCR product, $400 \mathrm{bp}$ ). The transcribed cDNA was mixed with each primer and Taq DNA polymerase (Takara, Japan), and then amplified. PCR was performed as follows: after incubation at $94^{\circ} \mathrm{C}$ for $5 \mathrm{~min}$, Stat 3 , MMP- 9 and $\beta$-actin underwent 35 cycles of reaction $\left(94^{\circ} \mathrm{C}\right.$ for $30 \mathrm{sec}, 61^{\circ} \mathrm{C}$ of Stat $3 ; 58^{\circ} \mathrm{C}$ of MMP-9 and $\beta$-actin for $30 \mathrm{sec}$, $72^{\circ} \mathrm{C}$ for $40 \mathrm{sec}$ ). After cycling, the samples were incubated at $72^{\circ} \mathrm{C}$ for $10 \mathrm{~min}$. The PCR products were analyzed on $1.5 \%$ agarose gel containing GoldenView. Stained bands were visualized under UV light and were photographed.

Western blot analysis. 4T1 cells were harvested from 6-well plates at $80 \%$ confluency. Cells were washed with cold PBS and lysed with cell lysis buffer containing protease inhibitor (Beyotime). Whole cell lysates were centrifuged at $13000 \mathrm{r}$ for $30 \mathrm{~min}$ at $4^{\circ} \mathrm{C}$. Protein concentrations were determined by the BCA reaction (Beytime Biotechnology, China). Proteins (50 $\mu \mathrm{g}$ of protein/lane) from control or treated cells were separated by SDS-PAGE and transferred to polyvinylidene difluoride membrane (PVDF) membranes with a Bio-Rad semi-dry transfer system (Bio-Rad). The membranes were blocked with $5 \%(\mathrm{v} / \mathrm{v})$ non-fat milk overnight and then incubated with primary antibody for $12 \mathrm{~h}$ at $4^{\circ} \mathrm{C}$. After washes, the membrane were incubated with appropriate antibody conjugated with horseradish peroxidase as secondary antibody for $1 \mathrm{~h}$ at $37^{\circ} \mathrm{C}$, followed by three washes with TBST (Tris-buffer saline containing $0.1 \%$ Tween 20). Observations of signals were obtained by enhanced chemiluminescence (ECL reagent, Millipore, USA) according to the manufacturer's instructions. Intensities of the bands obtained from the RT-PCR and western blot analysis were analyzed using the Quantity One software (Bio-Rad).

Invasion assay. The 4T1 cell invasive ability with or without treatment was examined using the membrane Transwell culture system. Transwell membrane $(8 \mu \mathrm{m}$ pore size, $6.5 \mathrm{~mm}$ diameter; Sigma) coated with Matrigel (Sigma) overnight at $4^{\circ} \mathrm{C}$ were used for the invasion assay. The $4 \mathrm{~T} 1$ cells from the control or treated groups after $48 \mathrm{~h}$ were trypsinized, centrifuged, and resuspended at $2.5 \times 10^{5} / \mathrm{ml}$ in DMEM without FBS. Then, cells $\left(2.5 \times 10^{4} /\right.$ well $)$ were seeded onto the upper chamber of pre-coated Transwell membranes. The lower chamber of the Transwells contained DMEM with 10\% FBS. The cells on the upper chamber and Matrigel coating the membranes were swabbed with Q-tip after $18 \mathrm{~h}$ incubation. Then, the membranes were fixed with $4 \%$ paraformaldehyde and stained with $0.1 \%$ crystal violet. The cells attaching to the lower surface of the polycarbonate filter were counted under light microscopy (magnification, x200). The experiments were performed in thrice in triplicate. The 4T1 cells obtained from the Transwell assay were analyzed using the Image $\mathrm{J}$ software (NIH, USA).
Migration assay. The 4T1 cells from the control or treated group were trypsinized and suspended in DMEM medium without FBS. Transwell membranes were used. Then, cells ( $2 \times 10^{4} /$ well) were seeded onto the upper chamber of Transwells. Lower wells of the Transwell chamber contained DMEM with $10 \%$ FBS as chemotactic medium. Then, the chamber was incubated in a humidified incubator for $3 \mathrm{~h}$. The medium was removed from the upper chamber and the filtered cells were fixed with $4 \%$ paraformaldehyde and stained with $0.1 \%$ crystal violet. The migrated $4 \mathrm{~T} 1$ cells were evaluated under the microscope, and random fields were scanned (five fields per filter) for the cells obtained at the lower membrane side only. The number of 4T1 cells obtained from the Transwell assay were analyzed using the Image $\mathbf{J}$ software.

Statistical analysis. The repeated-measures ANOVA test was used between mutiple comparisons analysis with the SPSS17.0 software. P-values $<0.05$ were taken to denote significant differences.

\section{Results}

Complex formation of PR39 with siRNA. In order to ascertain whether PR39 and siRNA could form a complex, $100 \mathrm{nM}$ scrambled siRNA was mixed with PR39 at different concentration ratios. As shown by a gel-shift assay PR39 was able to bind siRNA in a dose-dependent manner (Fig. 1A), and the 1:90 molar ratio of siRNA to PR39 was an optimal condition. We measured the cytotoxicity of PR39 usig the MTT assay (Fig. 1B), and observed that PR39 at $3 \mu \mathrm{M}$ to $36 \mu \mathrm{M}$ had no effect on the cell viability of 4T1 cells. These results indicate that PR39 could interact with siRNA and form complexes, and it was barely nontoxic to cells.

Cellular colocalization of the FITC-PR39/Cy3-siRNA complex. To determine whether PR39 could deliver siRNA into the cytoplasm, 4T1 cells were transfected with Cy3-labeled siRNA complexed to FITC-labeled PR39. After $6 \mathrm{~h}$ treatment, the intracellular colocalization of the double-labeled PR39/ siRNA complex in 4T1 cells was assessed by fluorescence microscopy (Fig. 1C).

Optimal silencing effect of PR39/Stat 3 siRNA. To detect the gene-silencing effect of the Stat3 siRNA delivered by PR39, 4T1 cells stably expressing high levels of Stat3 were transfected with Stat3-siRNA/PR39. Stat3 mRNA expression was determined after 24-72 $\mathrm{h}$ of incubation with an increasing molar ratio from 0:1 to 1:180 Stat3-siRNA/PR39. A maximum gene silencing effect assessed by mRNA and protein levels was observed after $48 \mathrm{~h}$ of treatment, and the optimal ratio of siRNA to PR39 was 1:90 (Fig. 2A). Lipo 2000/Stat3 siRNA was used as a positive control, and it seemed more potent than PR39/siRNA in silencing Stat3 expression (Fig. 2B and D).

Proliferation and cell cycle of $4 T 1$ cells is not affected by Stat 3 knockdown. Cell proliferation was measured by the MTT and FCM assays. The results suggest that the proliferation and cell cycle of 4T1 cells were not affected by Stat 3 knockdown (Fig. 3A and B). Cyclin D1 protein levels were not influenced by the Stat 3 reduction (data not shown). 


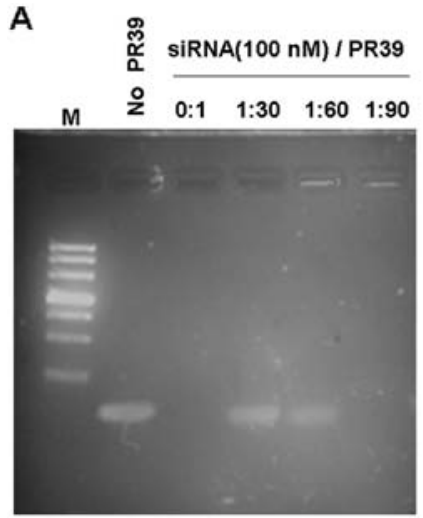

B
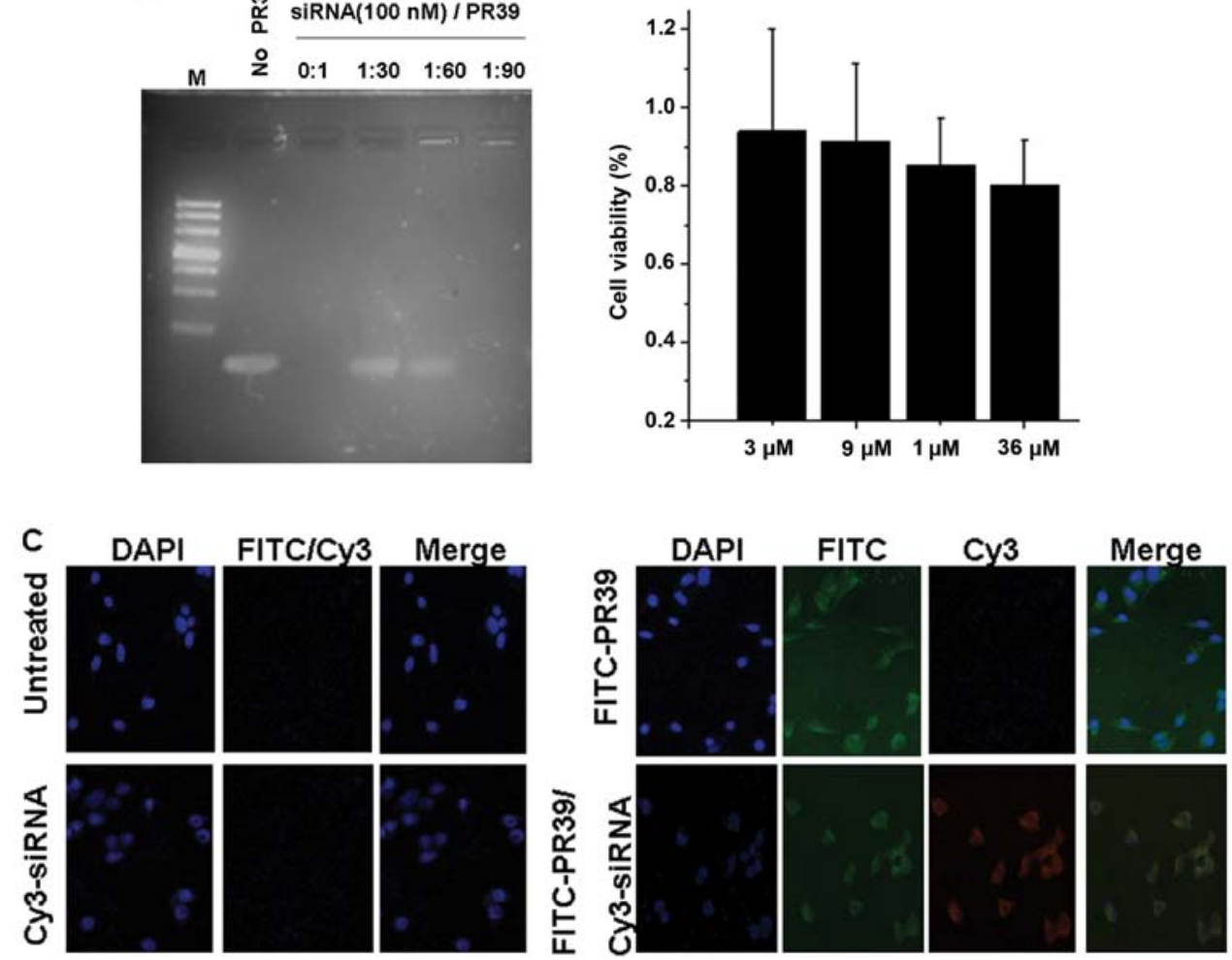

Figure 1. PR39 could formulate with siRNA and deliver siRNA to the 4T1 cells in vitro. (A) Gel shift assay of PR39/siRNA complex formation. It was shown that PR39 was able to bind siRNA in a dose-dependent manner, and 1:90 molar ratio of siRNA to PR39 was an optimal condition. (B) Cytotoxicity of PR39 at concentration from $3 \mu \mathrm{M}$ to $36 \mu \mathrm{M}$ for $4 \mathrm{~T} 1$ cells was evaluated by an MTT assay. Experiments were performed at least in triplicate each time; data shown are mean value and SD for three different measurements. PR 39 at concentration from $3 \mu \mathrm{M}$ to $36 \mu \mathrm{M}$ had no effect on the cell vitality for $4 \mathrm{~T} 1$ cells. (C) Intracellular localization of double-labeled PR39/siRNA complex in 4T1 cells by fluorescence microscopy and colocalization of PR39/siRNA after $6 \mathrm{~h}$ of treatment.

A

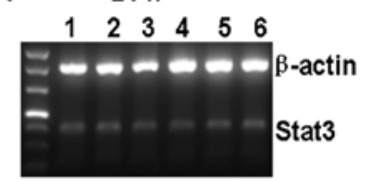

$48 \mathrm{~h}$

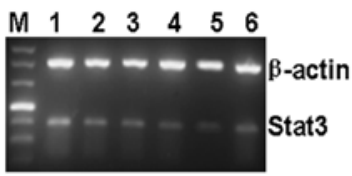

B

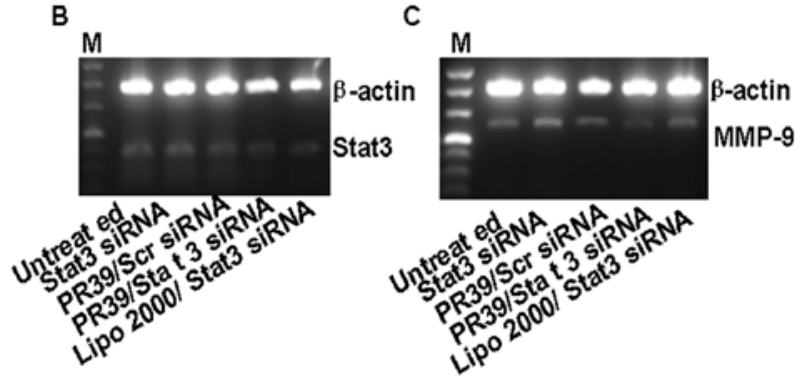

F

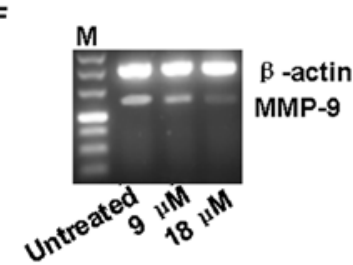

G

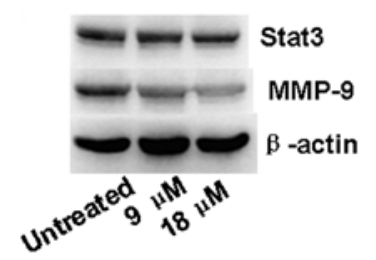

$\beta$-actin

un

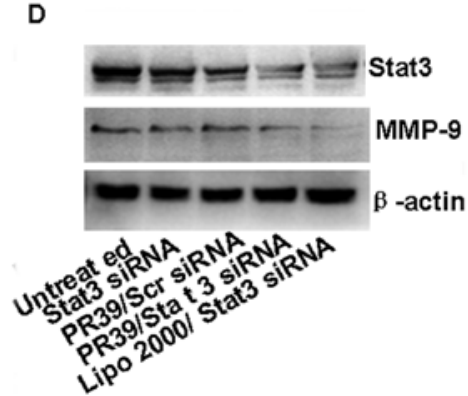

E

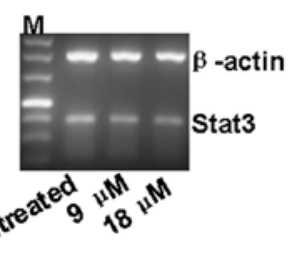

Figure 2. Gene silencing was analyzed by RT-PCR and Western blotting. (A) 4T1 cells stably expressing Stat 3 were treated with Stat 3 siRNA/PR39 of molar ratios ranging from 0:1 to 1:180, and Stat3 expression was analyzed at 24 or $48 \mathrm{~h}$. Lane 1, Untreated group; lane 2, $100 \mathrm{nM}$ Stat 3 siRNA; lane 3, Stat3 siRNA/ PR39 1:30; lane 4, Stat3 siRNA/ PR39 1:60; lane 5, Stat3 siRNA/ PR39 1:90; lane 6, Stat3 siRNA/PR39 1:180. The maximum gene silencing effect was found after $48 \mathrm{~h}$ of treatment, and the optimal ratio of siRNA to PR39 was 1:90. 4T1 cells were examined for (B) Stat3 mRNA and (D) protein levels after treatment with PR39/ Stat3 siRNA complex for $48 \mathrm{~h}$. Untreated group, Stat3 siRNA and PR39/Scr siRNA were used as negative control; Lipo 2000/Stat3 siRNA was used as a positive control. MMP-9 mRNA (C) and protein levels (D) were determined after treatment with the PR39/Stat3 siRNA complex for 48 h. Lipo 2000/ Stat3 siRNA, was used as a positive control, and seemed more potent than PR39/siRNA in silencing Stat 3 expression but had no effect on its downstream target molecular, MMP-9. (E-G) 4T1 cells were assayed for Stat3 and MMP-9 mRNA and protein levels after single PR39 treatment for $48 \mathrm{~h}$ assessing whether single PR39 could result in MMP-9 downregulation. 


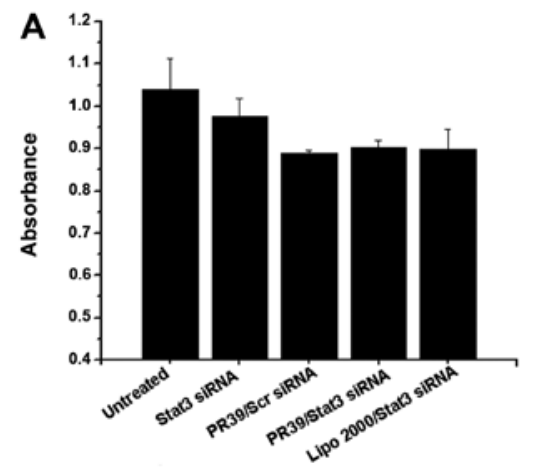

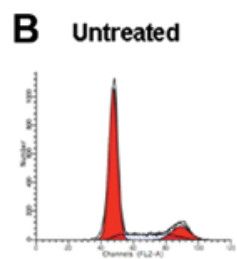

Diploid: $100.00 \%$ Dip $61: 71,41 \%$ st 47.40
Dip $62: 12.78 \%$ at 89.00 Dip G2: 12.78 \% at 89.00
Dip S: 15.81 \% $\quad$ G2/61: 1.89

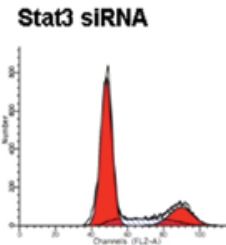

Diploid: $100.00 \%$ Dip $61: 67.48 \%$ at 48.00 Dip S: $15.76 \%$ \&2/G1: 1 .
PR39/Scr SIRNA

PR39/Stat3 siRNA

Lipo 2000/Stat3 siRNA

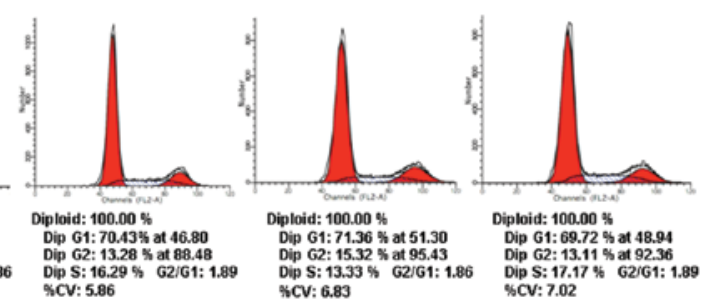

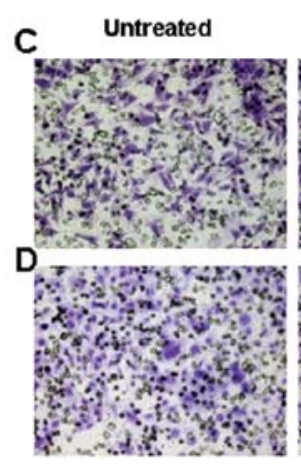

Stat3 siRNA

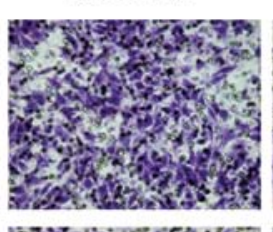

PR39/Scr SIRNA

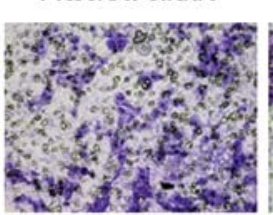

PR39/Stat3 sIRNA

Lipo 2000/Stat3 siRNA
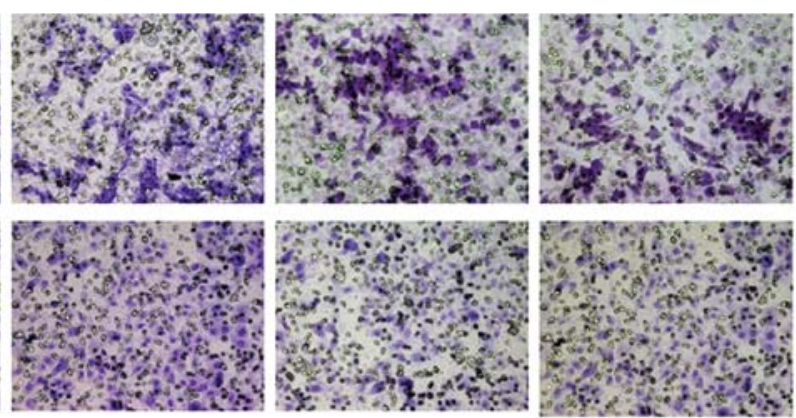
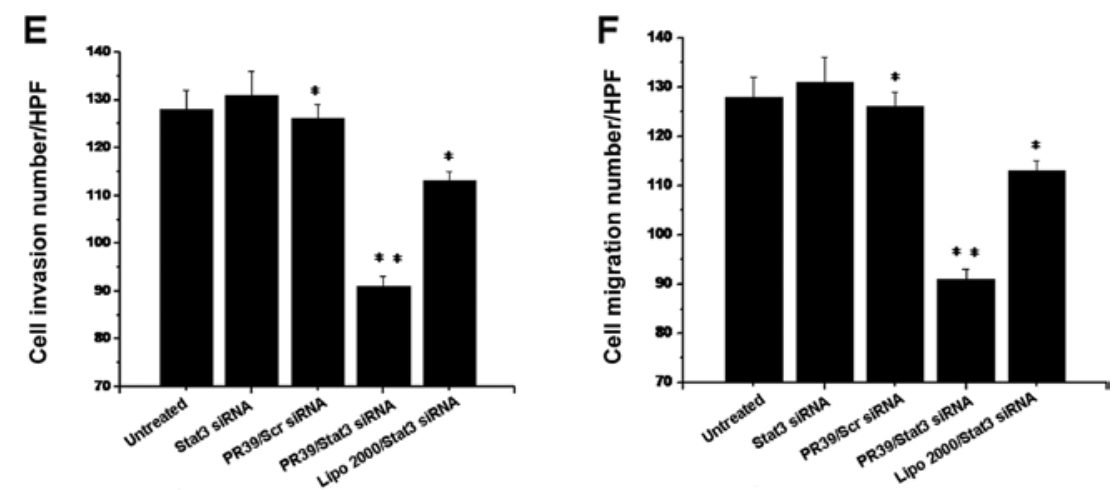
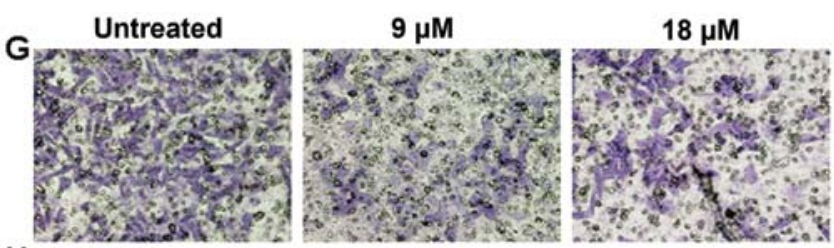

\section{H}

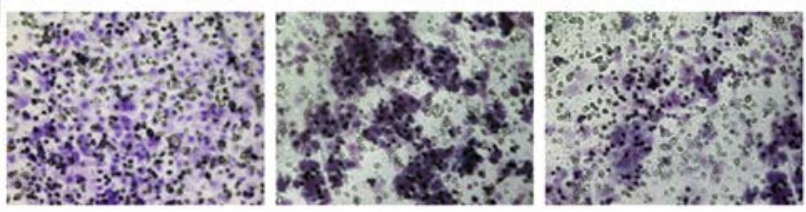

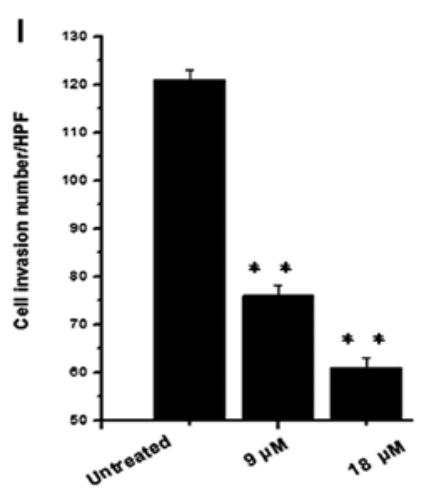

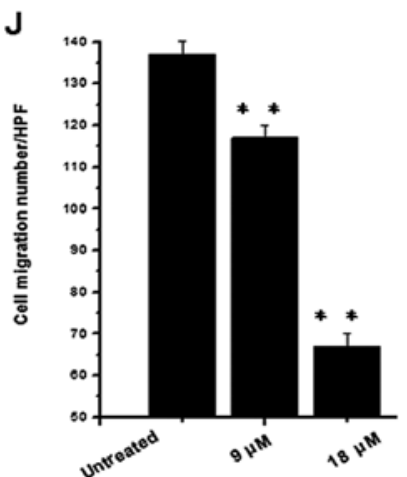

Figure 3. Cell proliferation, cell cycle, cell invasion and metastasis were analyzed after Stat3 knockdown in 4T1 cells. (A) Cell proliferation was examined using MTT assay. Each group was analyzed in triplicate. Reported data are the average of three separate experiments. (B) Cell cycle was analyzed by FCM assay in 4T1 cells treated by PR39/ Stat3 siRNA and Lipo 2000/ Stat3 siRNA compared with untreated, naked Stat3 siRNA, PR39/ Scr siRNA control group. These results suggested that proliferation and cell cycle of 4T1 cells were not affected by Stat3 knockdown. (C and D) Cell invasion and migration of 4T1 cells were investigated after treatment with various formulations by Transwell assay in vitro. The invaded and migrated cells were observed under microscope (Crystal Violet staining, x200). A representative example of the experimental result was shown. (E and F) Three independent experiments were performed. Columns: mean of invasive and migratory cell number from three independent experiments; bars: $\mathrm{SD} ;{ }^{*} \mathrm{P}<0.05,{ }^{* *} \mathrm{P}<0.01$, statistically significant. The cells treated with PR-39/Scr siRNA, PR-39/Stat3 siRNA and Lipo 2000/Stat3 siRNA were fewer significantly compared with naked Stat3 siRNA and untreated group. Although Lipo 2000/Stat3 siRNA displayed more potent compared to PR39/siRNA in silenced Stat3 expression, the cells invasive and migration capacity after being treated with Lipo 2000/Stat3 siRNA was not less compared to the PR39/siRNA group. (G and $\mathrm{H}$ ) The invasive and migration capacity of 4T1 cells were detected after treatment with single PR39 by Transwell apparatus in vitro. (I and J) The invaded and migrated cell number in $9 \mu \mathrm{M}$ and $18 \mu \mathrm{M}$ group was significantly lower than the untreated group. 
Inhibition of $4 T 1$ cell invasion and migration with PR39/ Stat 3 siRNA. The effect of Stat3 siRNA on the invasiveness and motility of 4T1 cells was determined using the Transwell assay. The cells treated with PR39/Scr siRNA, PR39/Stat3 siRNA were significantly fewer compared to the naked Stat3 siRNA and the untreated group (Fig. 3C-F). More interesting, although Lipo 2000/Stat3 siRNA displayed more potent activity than PR39/siRNA in silencing Stat3 expression, the invasive and migration capacity of the cells after treatment with Lipo 2000/Stat3 siRNA was not less than that of the PR39/siRNA group.

In addition, matrix matalloproteinase-9 (MMP-9) has been shown to be one of the Stat3 downstream target genes, which plays an important role in tumor cells invasion and migration. Therefore, MMP-9 expression was examined after $48 \mathrm{~h}$ transfection with PR39/Stat3 siRNA and Lipo 2000/Stat3 siRNA. These observations suggested that MMP-9 expression was significantly inhibited by the PR39/siRNA group compared to the Lipo 2000/Stat3 siRNA group (Fig. 2C and D). Given that previous reports demonstrated that PR39 gene transduction altered the invasive activity and actin structure in human hepatocellular carcinoma cells (26), we investigated the effect of PR39 alone on cell invasion and migration. As shown in Fig. 3G and J, PR39 (9 or $18 \mu \mathrm{M})$ could significantly inhibit 4T1 cell invasion and migration, and it was estimated that PR39 and Stat 3 siRNA could have a synergistic effect in the invasion and migration of $4 \mathrm{~T} 1$ cells.

\section{Discussion}

siRNA shows potential as a therapeutic tool against genes causing many diseases, but the effectiveness of these siRNAs are limited by their poor cellular permeability and insufficient stability profile. Currently, Lipofectamine transfection is one of the most popular methods of siRNA delivery into cell cytoplasm. However, the Lipofectamine transfection is confined to specific cell types and is toxic to cells and animals (27). In recent years, cell penetrating peptides have been effective carriers delivering siRNA in vitro. Specifically, an argininerich intracellular delivery peptide could noncovalently deliver macromolecules, and translocate into animal cells and tissues (28). For example, the nona-arginine (R9) was able to noncovalently deliver siRNA into mammalian cells (29), and efficient RNA interference was also obtained by an arginine peptide (R15) in vitro and in vivo (30). Zhang et al reported that human antimicrobial peptide LL-37 could deliver nucleic acids into the host cells as a cell-penetrating peptide (31). It is of interst to investigate whether with abundance of cationic amino acids, PR39 could be used as a novel siRNA carrier. Thus, we initially investigated the complex formation of PR39 with siRNA and its cellular colocalization. Then, we optimized the ratio of the PR39/siRNA complex, cell/complex incubation period and the concentration of siRNA. The results suggest that PR39 could form a complex with siRNA and translocate siRNA into 4T1 cells. The optimal ratio of siRNA to PR39 was 1:90 with a maximum gene silencing effect observed after $48 \mathrm{~h}$ treatment. Except for mice breast cancer cells, the cellular localization by PR39/siRNA appeared in HBL100, Hep3B and K562 cells (data not shown). These results showed that cationic PR39 could noncovalently contact with negative siRNA and translocate the complex into the cell cytoplasm. At present, the mechanisms underlying the cellular translocation of PR39 are poorly understood. It has been deduced that electrostatic attraction plays an important role in the strong binding and interaction with cancer cell membranes, caused by the interaction between the negatively charged component of the cancer cell surface and the positively charged cationic antimicrobial peptides (32).

To investigate the knockdown gene efficiency of PR39mediated siRNA, Stat3 siRNA/PR39 was introduced to 4T1 cells, and Stat 3 mRNA and protein levels were determined with RT-PCR and western blot analysis. As indicated in our studies, the Stat 3 siRNA/PR39 complex silenced approximately 50\% of the gene expression of Stat3, and the expression knockdown lasted for 72 h. However, approximately $70 \%$ gene reduction was observed with Lipo 2000 transfection used as a positive control (Fig. 2B and D). Lipo 2000/Stat3 siRNA displayed more potent activity than the PR39/siRNA in silencing Stat3 expression. Veldhoen et al also reported that a novel carrier peptide termed MPG $\alpha$ mediated delivery of siRNA might be less efficient compared with Lipo 2000 (33).

In order to further examine the influence of Stat 3 gene silencing on malignant biological features of tumor cells, 4T1 cells proliferation, cell cycle, invasion and migration were investigated. The results suggested that Stat 3 knockdown could not result in 4T1 cell proliferation inhibition and cell cycle arrest (Fig. 3A and B), while invasion and migration of 4T1 cells was strongly inhibited (Fig. 3C-F). Notably, although Lipo 2000/Stat3 siRNA displayed more potent activity than PR39/stat3 siRNA in silencing Stat3 expression, the PR39/ Stat3 siRNA complex showed a stronger suppression of 4T1 cell invasion and migration along with MMP-9 expression. Besides, PR39/Scr siRNA complex also demonstrated stronger suppression in cell invasion and migration, compared with naked siRNA. We hypothesized that single PR39 may play a role in cell invasion and migration. We present evidence that single PR39 inhibited invasive and migration activity of 4T1 cells and on the reduction of MMP-9 expression (Fig. 2E-G, Fig. 3G-J). Previous studies also reported that PR39 could alter the invasive activity and actin structure in human hepatocellular cancer cells (26). Therefore, it was shown that PR39 and Stat 3 siRNA could play synergistic roles in the invasion and migration of 4T1 cells (Fig. 3C-F).

In conclusion, we used PR39 as a novel siRNA delivery system, which could interact with siRNA, form complexes, and mediate delivery of siRNA into the cytoplasm to silence the target gene. The results also suggest that in addition to its anticarcinogenic activity, single PR39 may play a role in cell invasion and migration. PR39 and Stat 3 siRNA displayed synergistic biological effects on inhibiting cell invasion and migration of 4T1 cells, which were more dominent compared to the Lipo 2000 delivery system. Although further evidence is required to determine the exact mechanisms, our study highlights the potential of PR39 to mediate siRNA to the 4T1 cells.

\section{Acknowledgements}

We thank Dr Yang'an Wen at the First Affiliated Hospital, Chongqing Medical University for providing technical support. This work was supported by a grant from National Natural 
Science Foundation of China (no. 30971131); and by a grant from the Foundation of National Key Discipline in Laboratory Medicine (no. 2010104). This study was supported in part by the Key Laboratory of Diagnostic Medicine Designated by the Ministry of Education, Chongqing Medical University.

\section{References}

1. Yang SY: Sniper the hyperplasia of mammary glands and escort for the women health. Med People: 34-35, 2007.

2. Jun JY, Griffith JW, Bruggeman R, et al: Effects of polyamine depletion by alpha-difluoromethylornithine on in vitro and in vivo biological properties of 4T1 murine mammary cancer cells. Breast Cancer Res Treat 107: 33-40, 2008

3. Garcia R, Yu CL, Hudnall A, et al: Constitutive activation of Stat 3 in fibroblasts transformed by diverse oncoproteins and in breast carcinoma cells. Cell Growth Differ 8: 1267-1276, 1997.

4. Yu H and Jove R: The STATs of cancer - new molecular targets come of age. Nat Rev Cancer 4: 97-105, 2004.

5. Frank DA: STAT3 as a central mediator of neoplastic cellular transformation. Cancer Lett 251: 199-210, 2007.

6. Darnell JE Jr: Transcription factors as targets for cancer therapy Nat Rev Cancer 2: 740-749, 2002.

7. Chiu WT, Lee HT, Huang FJ, et al: Caveolin-1 upregulation mediates suppression of primary breast tumor growth and brain metastases by stat3 inhibition. Cancer Res 71: 4932-4943, 2011.

8. Blaskovich MA, Sun J, Cantor A, et al: Discovery of JSI-124 (cucurbitacin I), a selective Janus kinase/signal transducer and activator of transcription 3 signaling pathway inhibitor with potent antitumor activity against human and murine cancer cells in mice. Cancer Res 63: 1270-1279, 2003

9. Leong PL, Andrews GA, Johnson DE, et al: Targeted inhibition of Stat 3 with a decoy oligonucleotide abrogates head and neck cancer cell growth. Proc Natl Acad Sci USA 100: 4138-4143, 2003.

10. Meydan N, Grunberger T, Dadi H, et al: Inhibition of acute lymphoblastic leukaemia by a Jak-2 inhibitor. Nature 379: 645-648, 1996.

11. Mora LB, Buettner R, Seigne J, et al: Constitutive activation of Stat 3 in human prostate tumors and cell lines: direct inhibition of Stat 3 signaling induces apoptosis of prostate cancer cells. Cancer Res 62: 6659-6666, 2002

12. Nakajima K, Yamanaka Y, Nakae K, et al: A central role for Stat3 in IL-6-induced regulation of growth and differentiation in M1 leukemia cells. EMBO J 15: 3651-3658, 1996.

13. Ni Z, Lou W, Leman ES, et al: Inhibition of constitutively activated Stat 3 signaling pathway suppresses growth of prostate cancer cells. Cancer Res 60: 1225-1228, 2000

14. de Fougerolles A, Vornlocher HP, Maraganore J and Liberman J: Interfering with disease: a progress report on siRNA-based therapeutics. Nat Rev Drug Discov 6: 443-453, 2007.

15. Akhtar S and Benter I: Toxicogenomics of non-viral drug delivery systems for RNAi: potential impact on siRNA-mediated gene silencing activity and specificity. Adv Drug Deliv Rev 59: 164-182, 2007

16. Bumcrot D, Manoharan M, Koteliansky V and Sah DW: RNAi therapeutics: a potential new class of pharmaceutical drugs. Nat Chem Biol 2: 711-719, 2006.
17. Nakamura Y,Kogure K,Futaki S and Harashima H: Octaargininemodified multifunctional envelope-type nano device for siRNA. J Control Release 119: 360-367, 2007.

18. Agerberth B, Lee JY, Bergman T, et al: Amino acid sequence of PR-39. Isolation from pig intestine of a new member of the family of proline-arginine-rich antibacterial peptides. Eur J Biochem 202: 849-854, 1991.

19. Boman HG, Agerberth B and Boman A: Mechanisms of action on Escherichia coli of cecropin P1 and PR-39, two antibacterial peptides from pig intestine. Infect Immun 61: 2978-2984, 1993.

20. Storici $P$ and Zanetti M: A cDNA derived from pig bone marrow cells predicts a sequence identical to the intestinal antibacterial peptide PR-39. Biochem Biophys Res Commun 196: 1058-1065, 1993.

21. Shi J, Ross CR, Leto TL and Blecha F: PR-39, a proline-rich antibacterial peptide that inhibits phagocyte NADPH oxidase activity by binding to Src homology 3 domains of $\mathrm{p} 47 \mathrm{phox}$. Proc Nat Acad Sci USA 93: 6014-6018, 1996.

22. Chan YR and Gallo RL: PR-39, a syndecan-inducing antimicrobial peptide, binds and affects p130(Cas). J Biol Chem 273: 28978-28985, 1998

23. Gao Y, Lecker S, Post MJ, et al: Inhibition of ubiquitinproteasome pathway-mediated I kappa B alpha degradation by a naturally occurring antibacterial peptide. J Clin Invest 106: 439-448, 2000.

24. Alshamsan A, Hamdy S, Samuel J, et al: The induction of tumor apoptosis in B16 melanoma following STAT3 siRNA delivery with a lipid-substituted polyethylenimine. Biomaterials 31: 1420-1428, 2010

25. Ye Y, Yin DT, Chen L, et al: Identification of Piwil2-like (PL2L) proteins that promote tumorigenesis. PLoS One 5: e13406, 2010.

26. Ohtake T, Fujimoto Y, Ikuta K, et al: Proline-rich antimicrobial peptide, PR39 gene transduction altered invasive activity and actin structure in human hepatocellular carcinoma cells. Br J Cancer 81: 393-403, 1999

27. Ohki EC, Tilkins ML, Ciccarone VC and Price PJ: Improving the transfection efficiency of post-mitotic neurons. J Neurosci Methods 112: 95-99, 2001.

28. Wang $\mathrm{YH}$, Chen $\mathrm{CP}$, Chan $\mathrm{MH}$, et al: Arginine-rich intracellular delivery peptides noncovalently transport protein into living cells. Biochem Biophys Res Commun 346: 758-767, 2006.

29. Wang YH, Hou YW and Lee HJ: An intracellular delivery method for siRNA by an arginine-rich peptide. J Biochem Biophys Methods 70: 579-586, 2007.

30. Kim SW, Kim NY, Choi YB, et al: RNA interference in vitro and in vivo using an arginine peptide/siRNA complex system. J Control Release 143: 335-343, 2010.

31. Zhang X, Oglecka K, Sandgren S, et al: Dual functions of the human antimicrobial peptide LL-37-target membrane perturbation and host cell cargo delivery. Biochim Biophys Acta 1798: 2201-2208, 2010.

32. Hoskin DW and Ramamoorthy A: Studies on anticancer activities of antimicrobial peptides. Biochim Biophys Acta 1778: 357-375, 2008.

33. Veldhoen S, Laufer SD, Trampe A and Restle T: Cellular delivery of small interfering RNA by a non-covalently attached cell-penetrating peptide: quantitative analysis of uptake and biological effect. Nucleic Acids Res 34: 6561-6573, 2006. 